\title{
Robot Aided Remote Medical Assistance System using LabVIEW
}

\author{
N Natarajan \\ Student, E \& I, \\ SRM University \\ Kattankulathur-603203
}

\author{
S Aparna \\ Student, E \& I, \\ SRM University \\ Kattankulathur-603203
}

\author{
J Sam Jeba Kumar \\ Assistant Professor, ICE Dept., \\ SRM University \\ Kattankulathur-603203
}

\begin{abstract}
The medical treatment and diagnosis of patients in India especially in rural regions are adversely affected due to lack of proper healthcare and a poor patient to doctor ratio. This paper attempts to bring out the concept of cost effective implementation of a robotic doctor for performing advanced telemedicine. The robot designed here assists doctor to carry out medical tests and provides patient-doctor interaction in case of physical absence of the doctor near the patient.
\end{abstract}

\section{General Terms}

Medibot, Development of wireless communication, Integrated hardware design and implementation.

\section{Keywords}

ECG, EEG, NI ELVIS, LabVIEW ${ }^{\mathrm{TM}}$ platform, Advanced Telemedicine, National Instruments - Single Board Reconfigurable Input Output (NI-SbRIO).

\section{INTRODUCTION}

In this modern era, robots perform an important role in making healthcare and medical technologies more accessible and affordable to people. Robot aided healthcare system is much more reliable, sustainable, accurate and beneficial to people than the conventional systems.

A poor patient to doctor ratio and inaccessibility to advanced healthcare systems in a large part of the country and the world are acute problems that the medical fraternity faces today. Robots have been tested and tried to solve these problems and the effort has been successful as robotic applications have evolved from simple day to day activities like cleaning and washing to performing critical surgeries on the heart and the brain.

Robotics in medical application and healthcare has provided impressive remarks for research on developing systems that provide support to mankind. Personalized care, quality and efficiency of the robots are few worthy deliverables of their potential in the technologically developing world.

\section{THE CHALLENGE}

In the present technology [1], many outsets have arisen where Electrocardiography (EEG) and Electroencephalography (EEG) have been designed and optimized under different medical circumstances. The most conventional tradeoffs among all of them can be on the software and hardware forefront which stood ahead as the medical world demands compactness and proficiency in performance.
The challenge faced here, explains the development of a robot which would be used for analysing [2] medical tests like ECG, EEG and pulse rate measurement carrying out at the patient's location. The doctor has no information about the patient's health condition when he is not physically present near the patient. The robotic system developed herein acts as a medium of communication [3] between the patient and the doctor in case of physical absence of the doctor and also performs medical tests like ECG, EEG and pulse rate measurement when asked by the doctor to do so.

\section{LabVIEW ${ }^{\mathrm{TM}}$ ENVIRONMENT}

Laboratory Virtual Instrument Engineering Workbench $\left(\mathrm{LabVIEW}^{\mathrm{TM}}\right)$ is one of the most widely used graphical programming languages for developing applications ranging from small simulation of logics to a standalone embedded platform application. The software package interacts with user friendly hardwares from National Instruments as well as with other third party hardwares.

LabVIEW ${ }^{\mathrm{TM}}$ provides the feature of expanding the application level without compromising on the hardware support by developing reconfigurable hardwares and peripherals which offer high flexibility to implement complex algorithms especially while developing standalone systems.

LabVIEW $^{\mathrm{TM}}$ is easy to learn and has very less work on conventional coding. The time for implementing an application compared to conventional coding is very less which contributes enormously for an industry to test, measure and control an industrial process effectively. The files developed are called Virtual Instruments (VIs).

\section{SYSTEM CONFIGURATION}

The system developed consists of the following components:

- ECG and EEG hardware (in-house developed).

- Pre-amplifier circuits [4].

- ECG and EEG measurement electrodes [5], [6].

- Power supply unit.

- Camera.

- National Instruments Educational Laboratory Virtual Instruments Suite I (NI-ELVIS I) for the testing of individual components.

- $\quad$ NI SbRIO 9642 for the final implementation 


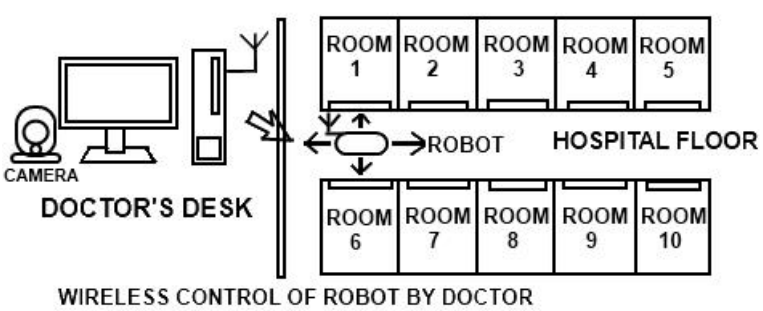

Fig.1.The layout illustrating the working of Robodoc.

To improve the cause of medical treatment, a robotic system is developed herein using NI LabVIEW ${ }^{\mathrm{TM}}$. The robot is under the supervisory control of the doctor. The doctor controls and commands the robot. The doctor makes the robot move to a particular patient's room and monitors the patient's room through high resolution camera. These images can be viewed continuously by the doctor and the doctor also interacts with the patient through the camera. The robot can also be commanded by the doctor to perform medical tests like ECG, EEG and pulse rate measurement. The reports of these tests would be sent to the doctor's computer wirelessly through Transmission Control Protocol/Internet Protocol (TCP/IP) protocol. The doctor then analyses these reports and detects abnormalities using LabVIEW $^{\mathrm{TM}}$. Graphical system design approach solves the problem by enabling user friendly interface. The doctors would find it easy to operate the system and analysis of reports would also be easy.

The doctor would control the robot's X-Y axis movements through LabVIEW ${ }^{\mathrm{TM}}$. Twelve lead ECG [7] analysis are performed here in order to determine the accuracy and the test bed software requirement for validation of results. The robot conducts ECG testing with the aid of nurse on the patient, when commanded by the doctor to do so and [11] sends the test report to the doctor's computer. The software is programmed to detect abnormalities in the reports arising due to the patient's health condition and report the abnormalities. The software also calculates the patient's pulse rate from the ECG report.

The LabVIEW ${ }^{\mathrm{TM}}$ software at the robot end is programmed to conduct EEG testing. The software is programmed to compare these reports with the standard Delta and Beta waves generated in the EEG testing of a healthy person and detect abnormalities [10], if any.

An interactive robot system with its functionalities in control of the doctor provides doctors with an enhanced concept of telemedicine.

\subsection{Hardware Development and Testing}

Developing hardware for measurement of ECG and EEG requires very high precision, satisfying the industrial performance standards. Reduction in cost of system developed, gaining experience in developing hardware for test-bench were one of the few reasons for the in-house development.

The circuits designed were tested rigorously in order to meet better accuracy during measurement of real time signals. The simulation of the circuit was carried out to ensure that the gains, Common Mode Rejection Ratio (CMRR), Grounding and Resonant frequency were taken care of in the initial phase itself.

The signals acquired from the body are amplified by the signal conditioning circuit on the hardware. The signals are then filtered and sent to the NI ELVIS kit. The software processes the signal and analyses it for the purpose of detection of abnormalities, if any.

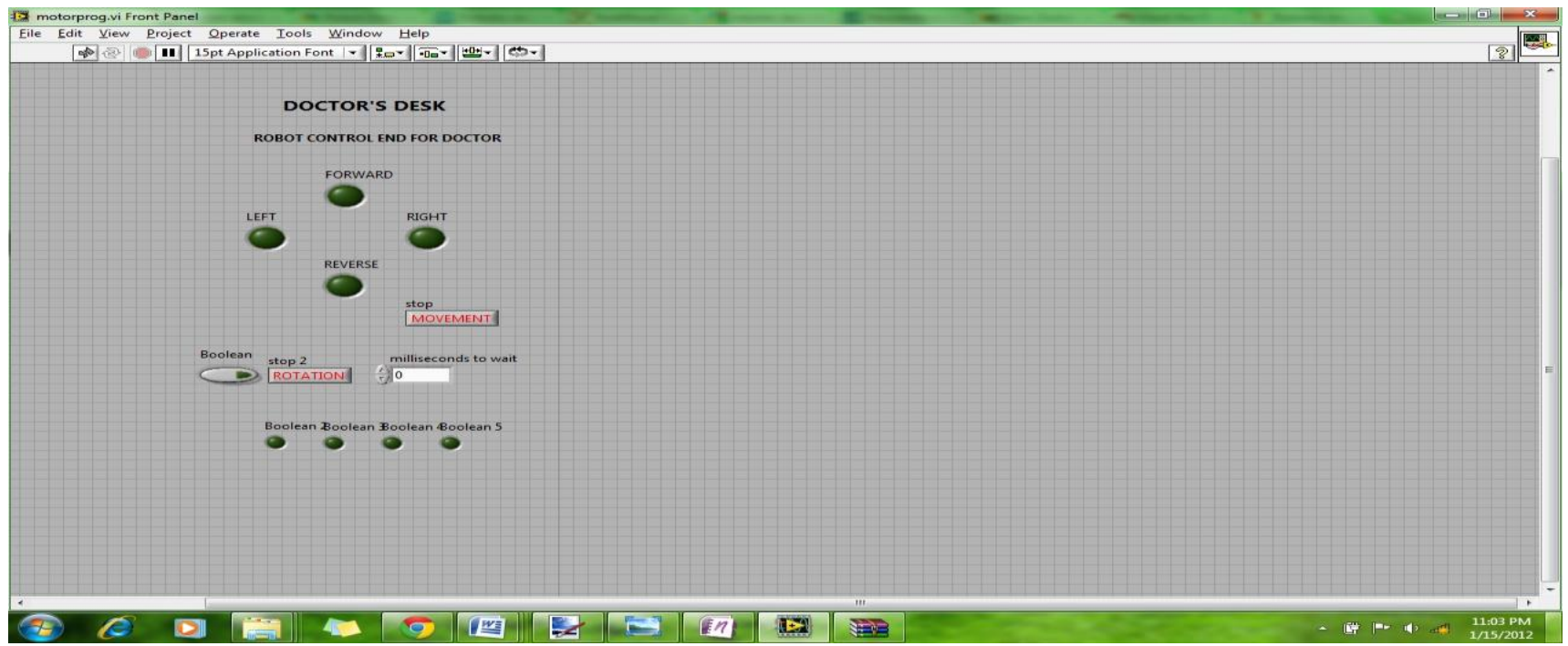

Fig.2.The front panel of robot control available at doctor's desk. 


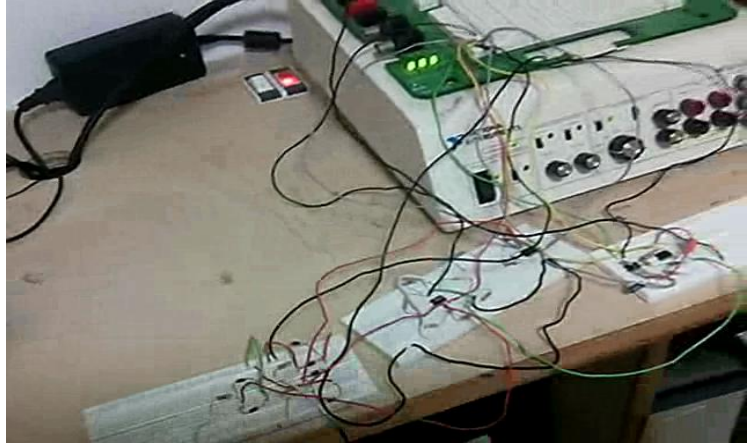

Fig.3. The hardware setup for signal acquisition with NI ELVIS I.
The front panel shown in figure 4 illustrates the observations made during the testing of a patient for the ECG [13]. The program intends to maintain a database of the persons undergoing testing recommended by the doctor. The development of this prototyping includes a report generation at the user interface to be filed for reference too. Thus different displays are provided to enhance the scanning ability of the signal received and for identification of symptoms.

The block diagram illustrated in figure 5 is a subvi program which is executed for computational organisation to identify the physical relationships of each of the [12] wavelets. The noise frequency of ac supply which is considered from the prototyping point of view was tackled in the real-time interfacing.

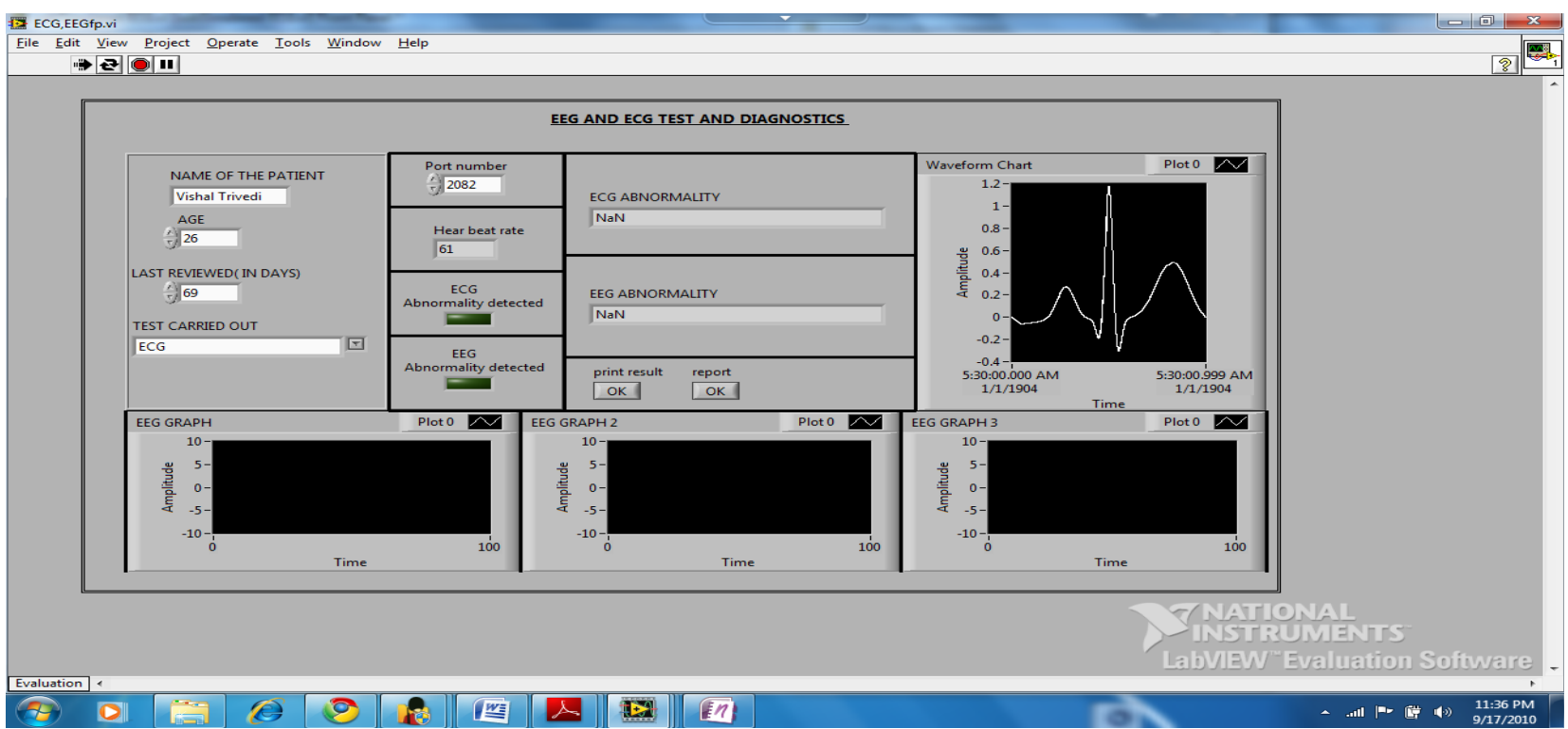

Fig.4.The front panel of the EEG and ECG testing setup.

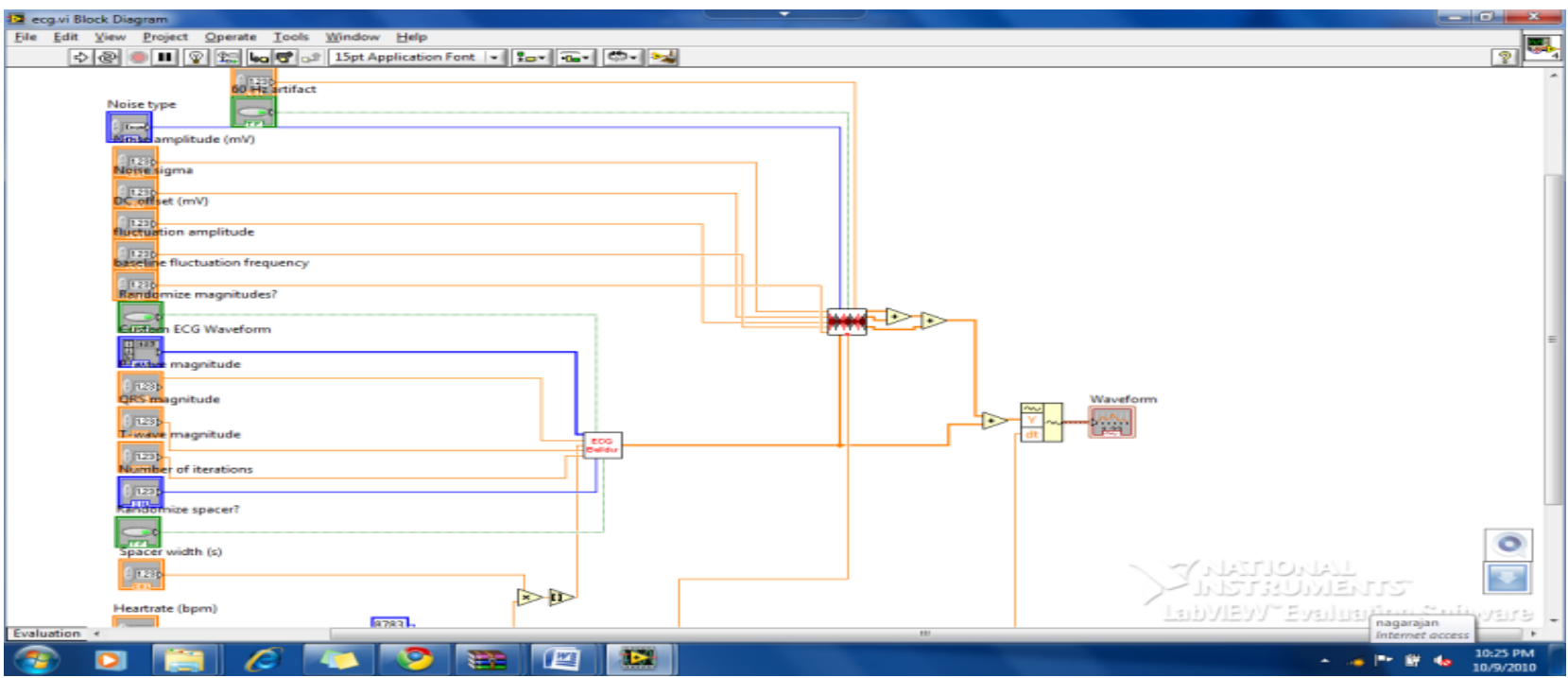

Fig.5. The block diagram of the sub vi developed for the ECG development 


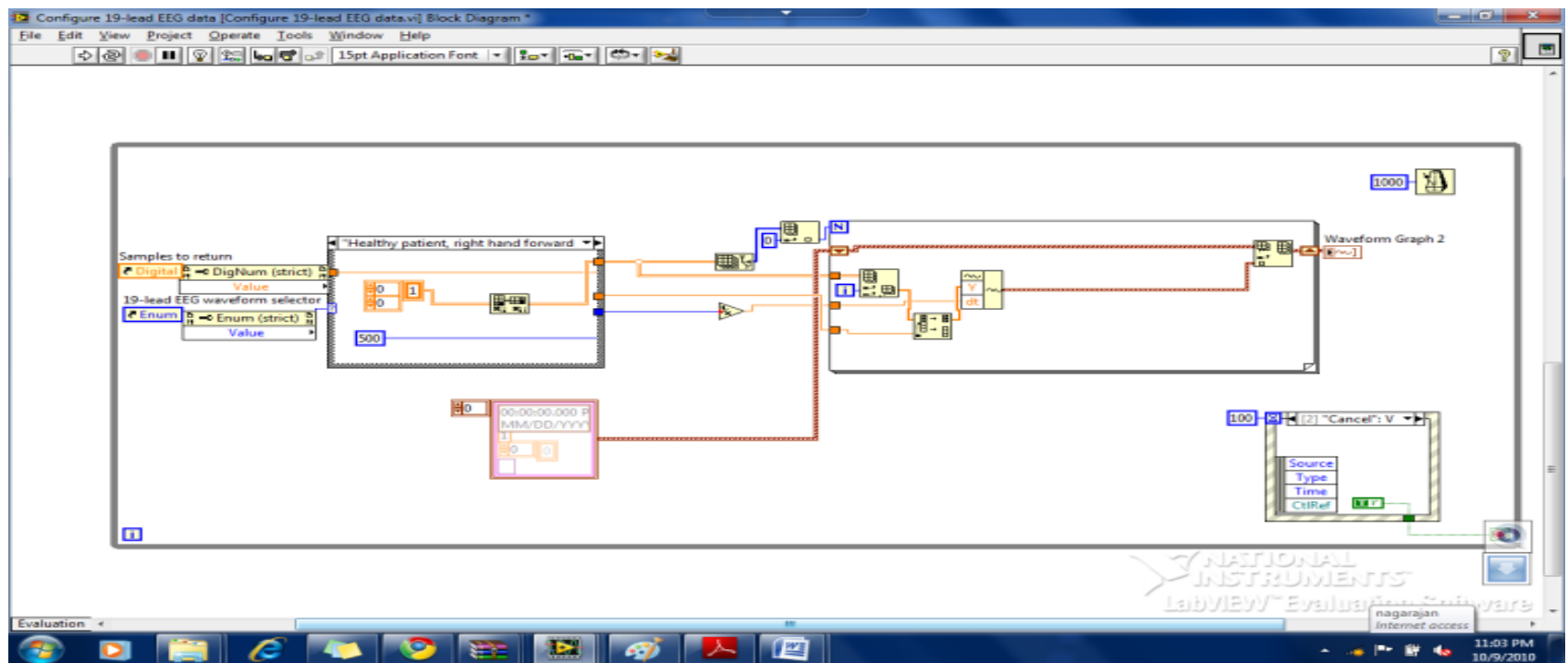

Fig.6.The Block diagram illustrating the EEG analysis.

The block diagram shown in figure 6 explains a subvi which selects the human EEG [14] signal range and determines the output analysis to be carried out. As seen in figure 6, the EEG analysis can't be affirmed with a single lead and hence a provision for three outputs to be read at a time is provided so as to extricate flaws in the judgement.

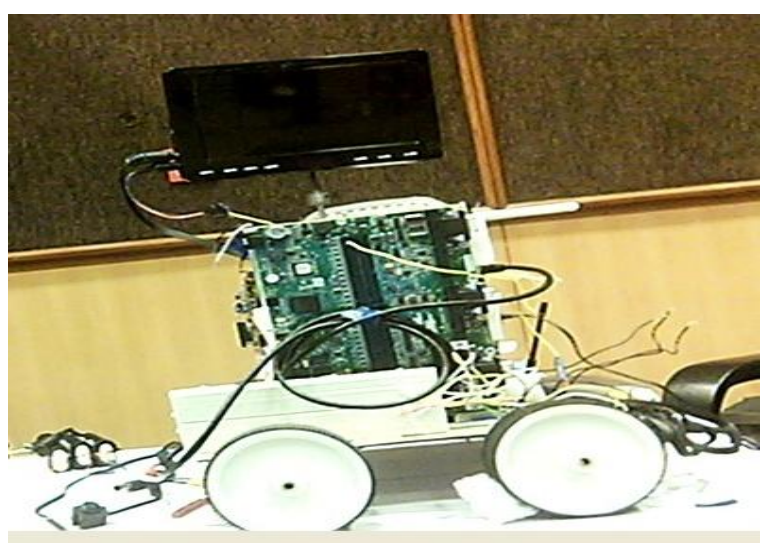

Fig.7.The robot prepared for implementing the ECG and EEG hardware and code designed using NI Sb-RIO 9642.

\section{IMPLEMENTATION}

The development and testing was carried out on NI-ELVIS kit. NI-SbRIO 9642 was used for developing the standalone robotic system. The implementation phase required integration of all hardwares onto the mechanical structure. The software code written as VI is converted into an embedded code suited for deployment in SbRIO-9642.

\section{CONCLUSION}

Camera aided interaction between the doctor and the patient provided by the robotic system supports the finding that patients prefer to talk to their regular physician, even if it is through the robot, rather than getting treated by an unknown doctor. Through this system, the doctor can do everything but touch the patient. The doctor can also interact with the nurses and other surgeons and doctors in case of emergency and give them instructions on the required immediate treatment for the patient. As an enhancement over the Robodocs [15], [16] launched recently in Dubai and New Zealand [17], this system can perform accurate measurements of ECG, EEG and pulse rate and also make the test reports available at the doctor's desk immediately. By employing this robot aided medical system, infection caused by human intervention in hospitals can be reduced considerably.

The developed VIs were incorporated with a human interface to validate results to support a dedicated test bed for implementation at SRM University premises, which would bring in a revolutionary and agile system to reality encompassing and fulfilling the needs of the country.

\section{ACKNOWLEDGMENTS}

We wish to thank SRM University, Kattankulathur for funding the project. The project won National Level $5^{\text {th }}$ in National Instruments Embedded System Design Contest named "NI Yantra 2010". The project was displayed at Indian Science Congress held in January, 2011 at SRM University, Kattankulathur among delegates.

We are highly obliged to be a part of this project which endeavours students to have a broad spectrum of innovation irrespective of the branch being trained at.

\section{REFERENCES}

[1] Berke Erem (8323) and Serkan Yazıcı (10276), FALL 2009 - 2010. Project 2 Report for EL 473 - Biomedical Instrumentation.

[2] Christine Moran, Leslie Goldberg, Yuheng Chen, "Collecting and filtering live ECG signa 1", Connexions module: m18957, Version 1.1: Dec 17, 2008 1:56 am US/Central.

[3] Gurpinder Kaur, "Design and Development of Dual Channel ECG Simulator and Peak Detector", Department of Electrical and Instrumentation Engineering, Thapar Institute of Engineering \& Technology, Deemed University, Patiala - 147004,June 2006.

[4] Gaetano Gargiulo, Paolo Bifulco1,Mario Cesarelli, Mariano Ruffo, Maria Romano, Rafael A Calvo, Craig Jin, André van Schaik, "An ultra-high input impedance ECG amplifier for long-term monitoring of athletes", Dipartimento di Ingegneria Elettronica, Biomedicae delle 
Telecomunicazioni, Federico University of Naples, Italy; 2School of Electrical and Information Engineering, University of Sydney, Australia, 2 July 2010.

[5] Gerald Ulrich,Charité , Book of Psychiatric encephalography.

[6] Ivan W. Selesnick and C. Sidney Burrus, "Generalized Digital Butterworth Filter Design", IEEE Transactions on Signal Processing, Vol. 46, NO. 6 June 1998.

[7] Internet. Reference notes on ECG and EEG. http://iopscience.iop.org/1742-6596/76/1/012038

[8] Juha Virtanen, Aug 21, 2007. Shield arrangement for ECG Lead wires. US Patent, US 7,260,428 B2.

[9] Jeffrey Groeger, Saul Miodownik, 4,957, 109 Sept 1990. Electrocardiograph system.

[10] Mudit Jain, Snigdha Chaturvedi, Varun Mithal, "Detection of Abnormalities and Diseases in ECG Data", Term report, Dept of Computer Science and Engineering ,IIT Kanpur - 208016 INDIA, Nov 8, 2008.

[11] Robert Lin, Ren-Guey Lee, Chwan-LuTseng, Yan-Fa $\mathrm{Wu}$, Joe Air Jiang, "Design and implementation of wireless multi-channel EEG recording system and EEG clustering method", Biomedical Engineering-
Applications, Basis and Communications, Vol. 18,No 06 Dec 2006.

[12] Rahman Jamal, Mike Cerna, John Hanks. Designing Filters Using the Digital Filter Design Toolkit. National Instruments, Application Note 097.

[13] William H.Righter, 5,191,89 , March 1993. ECG Signal Conditioner Electronic Circuit Diagram.mht.

[14] William Tatum, Aatif Husain, SelimBenbadis, Peter Kaplan, MD. Handbook of EEG interpretation, Demos Medical Publishing.mht.

[15] Internet. Robot Doctors could be the future of Medicine http://www.nbcchicago.com/news/health/Robot-DoctorsCould-be-Future-of-Medicine 102161529. htm \#ixzz1jXrihxeu.

[16] Internet, Robotic doctor to make debut in ME. http://www.khaleejtimes.com/DisplayArticle08.asp?xfile $=$ data $/$ middleeast $/ 2011 /$ April $/$ middleeast_April463.xml\& section $=$ middleeast.

[17] Internet, New Zealand to use robo doctor to treat patients. http://www.techchee.com/2008/08/20/newzealand-to-use-robo-doctor-to-treat-patients. 